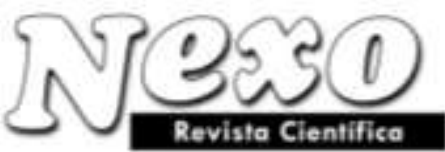

ISSN-E 1995-9516

Universidad Nacional de Ingeniería http://revistas.uni.edu.ni/index.php/Nexo http://dx.doi.org/10.5377/nexo.v30i01.5169

Vol. 30, No. 01, pp. 01-18/Junio 2017

\title{
CARGA SUPERFICIAL MÁXIMA EN LAGUNAS DE ESTABILIZACIÓN FACULTATIVAS DE NICARAGUA
}

\section{SURFACE LOADING RATE IN FACULTATIVE STABILIZATION PONDS FROM NICARAGUA}

\author{
Carlos M. Vanegas-Benavides; Rosa Verónica Reyes Rodríguez \\ Universidad Nacional de Ingeniería. Programa de Investigación, Estudios Nacionales y Servicios \\ Ambientales (PIENSA-UNI). Managua, Nicaragua. \\ carlos.vanegas@ vrid.uni.edu.ni; $\underline{\text { rosaveronica.reyes@hotmail.com }}$
}

(recibido/received: 15-Abril-2017; aceptado/accepted: 29-Mayo-2017)

\begin{abstract}
RESUMEN
Las lagunas de estabilización son una alternativa viable para el tratamiento de aguas residuales municipales, por ser un proceso natural, de bajo costo de operación y mantenimiento. En Nicaragua existen 17 Plantas de tratamiento por lagunas de estabilización, de las cuales 13 de ellas dan tratamiento a las aguas residuales de los municipios del pacífico de Nicaragua. Uno de los modelos más utilizados para el diseño de lagunas de estabilización es el de Carga Superficial Máxima, el cual contiene varios métodos de cálculo propuestos por diferentes autores. Sin embargo, en la normativa Nicaragüense no existen criterios suficientes que definan el método a emplear, por lo que cada diseñador tiene la libertad de utilizar el que mejor le parezca. Es por ello, que en la presente investigación se definió el método de cálculo de Carga Superficial Máxima que mejor se adapta a las plantas de tratamiento en estudio.
\end{abstract}

Palabras claves: Aguas residuales; Lagunas de estabilización facultativas.

\begin{abstract}
Stabilization ponds are a viable alternative for municipal wastewater treatment, as it is a natural, low-cost operation and maintenance process. In Nicaragua there are 17 treatment plants for stabilization ponds, of which 13 are given treatment to wastewater from the municipalities of Nicaragua Pacific. One of the most used models for the design of stabilization ponds is the Surface Loading Rate, which contains several calculation methods proposed by different authors. However, in the Nicaraguan legislation there are insufficient criteria to define the method to be used, so each designer is free to use as he pleases. It is for this reason that the present investigation defined the method of calculation of Surface Loading Rate that best adapts to the treatment plants under study.
\end{abstract}

Keywords: Wastewater; Facultative stabilization ponds. 
Carlos M. Vanegas-Benavides y Rosa Verónica Reyes Rodríguez

\section{INTRODUCCIÓN}

En la sociedad del conocimiento y la aldea global, los avances científicos y tecnológicos han permitido que en la actualidad exista una gran variedad de sistemas de tratamiento de aguas residuales. Ante esta realidad, algunos profesionales, de forma automática y sin pensarlo, desean adoptar las tecnologías de tratamiento de aguas residuales de los países industrializados, con la creencia generalmente errónea de que estas son las tecnologías apropiadas para implementar en los países en vías de desarrollo (Mara, 2004).

En el caso de Nicaragua, las lagunas de estabilización han demostrado ser la tecnología de tratamiento más apropiada para el tratamiento de aguas residuales municipales, debido a su eficiencia y bajos costos de operación y mantenimiento (Platzer, 2002). Del total de 35 plantas para el tratamiento de aguas residuales municipales, administradas por la Empresa Nicaragüense de Acueductos y Alcantarillados (ENACAL), 17 están conformadas por lagunas de estabilización, de las cuales 13 están ubicadas en la zona del pacífico de Nicaragua (PROATAS, 2013).

A pesar del amplio uso de las lagunas de estabilización, las guías técnicas nicaragüenses no especifican el modelo ni el método a utilizar para el diseño de lagunas de estabilización facultativa. Por el contrario, el documento "Guías técnicas para el diseño de alcantarillado sanitario y sistemas de tratamiento de aguas residuales" (INAA, 2004) en el numeral 11.12.7.1, da libertad al proyectista de escoger la correlación (modelo) a utilizar, tomando en cuenta las condiciones locales, recomendándole adoptar una carga de diseño menor a la determinada.

El problema de la afirmación antes mencionada radica en que existen diferentes modelos de correlación para diseñar lagunas de estabilización facultativas, siendo el más utilizado el modelo por Carga Superficial Máxima (CSM), del cual, se han propuesto una serie de métodos de cálculo que dependen de variables tales como geolocalización, radiación solar, temperatura del agua y temperatura ambiente. Sin embargo, al aplicar cada método bajo las mismas condiciones, se obtienen lagunas de estabilización con diferentes dimensiones.

Con base en lo antes mencionado, la presente investigación propone a los académicos y diseñadores de lagunas de estabilización de Nicaragua, la selección de un método de cálculo de carga superficial máxima, que permite un eficiente funcionamiento de remoción de materia orgánica y que a su vez optimiza áreas de terreno y recursos económicos.

\section{MODELO DE CARGA SUPERFICIAL MÁXIMA}

El diseño de un sistema de lagunas de estabilización es un proceso complejo, a pesar de la simplicidad, debido a que tiene carácter multidisciplinario y se debe tener extremo cuidado en seleccionar un modelo para establecer las dimensiones de las lagunas, aplicable a las condiciones climáticas del lugar (Cubillos Z, 2001). El diagrama de flujo más común de lagunas de estabilización es el constituido por una laguna primaria facultativa seguido de una laguna de maduración, ambas conectadas en serie (INAA, 2004).

Durante varias décadas se han propuesto numerosos modelos para el diseño de lagunas de estabilización facultativos, los cuales se pueden clasificar en Modelos racionales, Modelos matemáticos y modelos empíricos. Los modelos racionales ofrecen una explicación en términos cinéticos de lo que ocurre en las lagunas de estabilización. Por su parte, los modelos matemáticos son en realidad una subcategoría del modelo racional, con la diferencia que considera que las lagunas son sistemas dinámicos, con cinéticas complejas y regímenes no ideales de flujo. Finalmente, los modelos empíricos consisten en relaciones matemáticas sencillas deducidas de las observaciones experimentales realizadas en una determinada laguna

Nexo Revista Científica / Vol. 30, No. 01, pp. 01-18 / Junio 2017 
de estabilización, o en un grupo de ellos que trabajan en condiciones muy similares, tanto respecto a la climatología como a la alimentación.

El modelo de Carga Superficial Máxima (CSM) es el modelo empírico más utilizado y se ha considerado por varias décadas, como un importante criterio para el dimensionamiento de lagunas de estabilización facultativas, dejando atrás el criterio de oxígeno disuelto superficial como indicador de los procesos anaerobios (Yanez, 1981). El modelo consiste en determinar la carga máxima de materia orgánica por unidad de superficie ( $\mathrm{KgDBO} / \mathrm{ha}$ *d), que puede soportar una laguna en condiciones predominantemente facultativas. Los valores de CSM son inversamente proporcional al dimensionamiento de la laguna, de tal manera que, a menor CSM, se requiere de una mayor área de la laguna.

Cuando una laguna facultativa opera adecuadamente, ocurren simultáneamente procesos de reducción anaerobia, oxidación aerobia y reacción fotosintética. El límite entre el proceso de reducción y oxidación se llama oxipausa y varia de profundidad en dependencia de las horas del día: durante las mañana predomina el proceso aerobio y durante las noches predomina el proceso anaerobio. Sin embargo, cuando la CSM en una laguna de estabilización es superada, el proceso de reducción anaerobia predomina en la laguna tanto en el día como en la noche.

\section{LAGUNA FACULTATIVA}

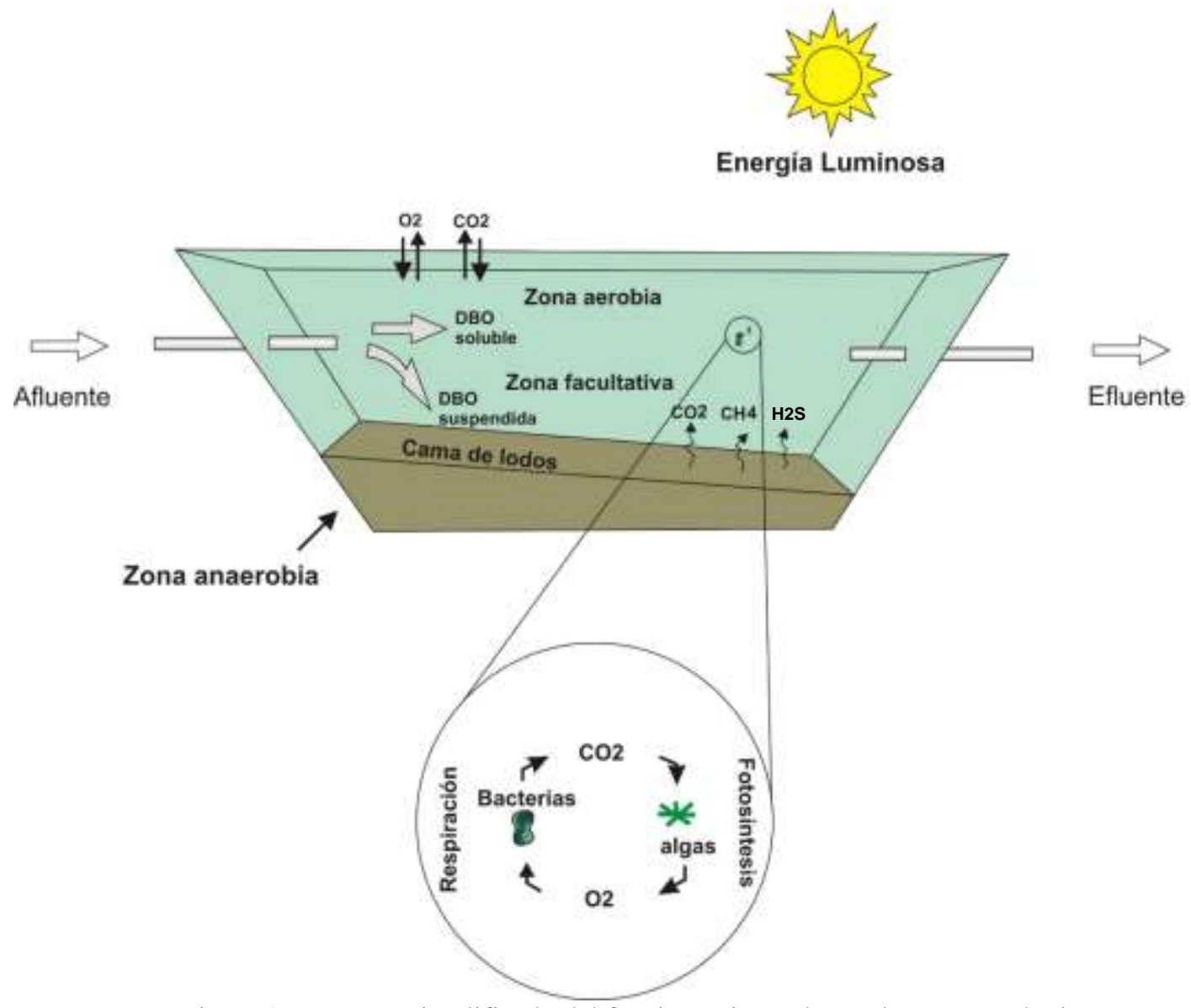

Figura 1. Esquema simplificado del funcionamiento de una laguna Facultativa. Tomado de (Von Sperling, 2007)

Nexo Revista Científica / Vol. 30, No. 01, pp. 01-18 / Junio 2017 
A lo largo de todo el mundo se han propuesto una serie de métodos empíricos de correlación para el cálculo de la Carga Superficial Máxima (CSM), utilizando como variables la latitud, la radiación solar, la temperatura ambiente y la temperatura del agua de la laguna. A continuación se presentan en la siguiente tabla los métodos más conocidos y empleados para el dimensionamiento de lagunas primarias facultativas.

Tabla 1. Métodos del Modelo de Carga superficial Máxima para el diseño de lagunas facultativas

\begin{tabular}{|c|c|c|c|}
\hline Método de diseño & Variable & Ecuación & Fuente de Información \\
\hline Método por & & & (Mara D. D., 1997) \\
\hline $\begin{array}{l}\text { Latitud } \\
\text { Soli J. Arceivala } \\
\text { (1970) }\end{array}$ & $\begin{array}{l}\text { Latitud Norte } \\
\quad 8-36^{\circ}\end{array}$ & $\lambda \mathrm{S}=375-6.25 \mathrm{~L}$ & \\
\hline Método por & Radiación & & (Rittmann \& McCarty, 2001) \\
\hline $\begin{array}{l}\text { Radiación Solar } \\
\text { (Rittmann \& } \\
\text { McCarty, 2001) }\end{array}$ & $\begin{array}{c}\text { solar } \\
\mathrm{KWh} / \mathrm{m} 2 * \mathrm{~d}\end{array}$ & $C S M=69.75 * R S$ & \\
\hline $\begin{array}{l}\text { Mc Garry \&Pescod } \\
\text { (1970) }\end{array}$ & $\mathrm{T}$ ambiente ${ }^{\circ} \mathrm{C}$ & $\lambda \mathrm{S}=60.26(1.0993) \mathrm{Ta}$ & $\begin{array}{l}\text { (Comisión Nacional del Agua, } \\
\text { 2007) }\end{array}$ \\
\hline $\begin{array}{l}\text { Duncan Mara } \\
\quad(1976)\end{array}$ & $\mathrm{T}$ ambiente ${ }^{\circ} \mathrm{C}$ & $\lambda S=20 \mathrm{~T}-120$ & $\begin{array}{l}\text { (Comisión Nacional del Agua, } \\
\text { 2007) }\end{array}$ \\
\hline Arthur (1983) & T ambiente ${ }^{\circ} \mathrm{C}$ & $\lambda \mathrm{S}=20 \mathrm{~T}-60$ & (Arthur, 1983) \\
\hline $\begin{array}{l}\text { Duncan Mara } \\
\quad(1987)\end{array}$ & T ambiente ${ }^{\circ} \mathrm{C}$ & $\lambda S=350(1.107-0.002 T)^{T-25}$ & (Mara D. D., 2004) \\
\hline México (2007) & T ambiente ${ }^{\circ} \mathrm{C}$ & $\lambda S=250(1.085)^{T-20}$ & $\begin{array}{c}\text { (Comisión Nacional del Agua, } \\
\text { 2007) }\end{array}$ \\
\hline $\begin{array}{l}\text { Fabián Yánez } \\
\text { (1984) }\end{array}$ & $\mathrm{T}$ del agua ${ }^{\circ} \mathrm{C}$ & $\mathrm{Lat}=357.4 * 1.085 \mathrm{~T}-20$ & (Yánez, 1984) \\
\hline $\begin{array}{l}\text { Norma OS.090 } \\
\text { Perú (2006) }\end{array}$ & $\mathrm{T}$ del agua ${ }^{\circ} \mathrm{C}$ & $C_{d}=250(1.05)^{T-20}$ & $\begin{array}{c}\text { (Ministerio de Transporte, } \\
\text { Comunicaciones, Vivienda y } \\
\text { Construcción, 2006) }\end{array}$ \\
\hline
\end{tabular}




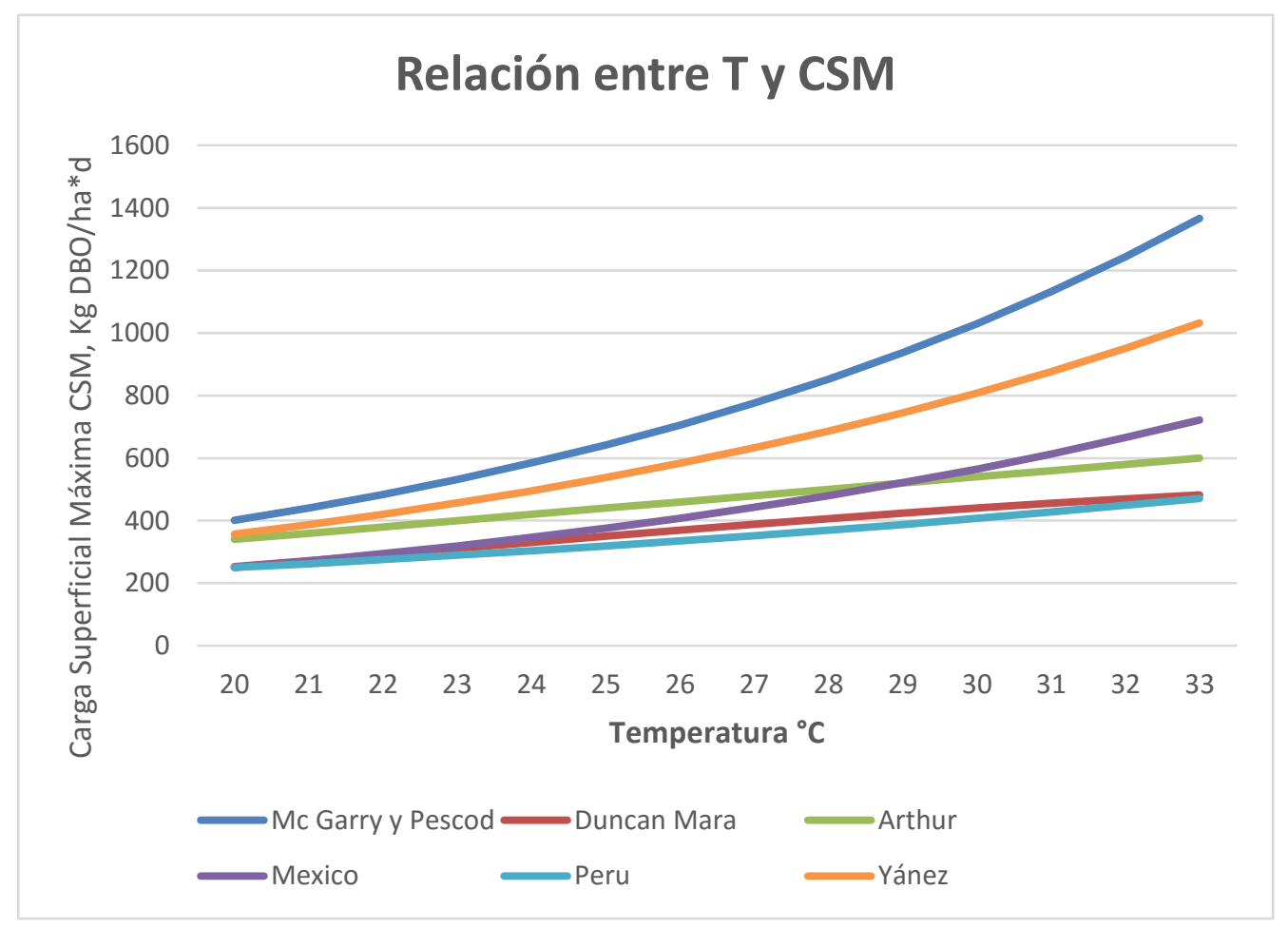

Figura 2. Relación entre la temperatura y la carga superficial en lagunas primarias facultativas. Fuente propia

La determinación de la Carga Superficial Máxima según la temperatura es el método empírico más utilizado en los países de clima cálido en América latina. En relación a este factor, se han propuesto una serie de correlaciones en función de la temperatura ambiente y la temperatura de la laguna, ambas en el mes más frío. Dichas correlaciones son producto de la observación de lagunas de estabilización en todo el mundo. Sin embargo, en sitios como la región pacífico de Nicaragua, donde la temperatura alcanza valores superiores a $30^{\circ} \mathrm{C}$, las fórmulas de correlación dan como resultado valores diferentes en los rangos de 400$1400 \mathrm{KgDBO} / \mathrm{ha}^{*} \mathrm{~d}$ (ver figura 2).

\section{INDICACIONES DE BUEN FUNCIONAMIENTO DE LAS LAGUNAS FACULTATIVAS}

La inspección visual de las lagunas de Estabilización proporciona información relativa a la forma en que están operando. Algunos de los indicadores cualitativos más importantes en el funcionamiento y estabilidad del sistema son el color, la observación al microscopio de los organismos y el olor.

La emisión de malos olores normalmente es causada por sobrecarga de la laguna, el aumento repentino en la carga orgánica, los cambios en la composición del agua residual o el desarrollo de condiciones anaerobias. Generalmente, provienen de los depósitos de lodo flotante y de la vegetación en putrefacción de la propia laguna.

Rolím (1999) afirma que una laguna facultativa o de maduración está funcionando adecuadamente cuando:

- El agua presenta una coloración verde intensa y está prácticamente libre de sólidos sedimentados. La coloración es más pálida para las lagunas de maduración. Las coloraciones verde - azuladas denotan la presencia de algas verdiazules (cianofíceas), que tienen efectos negativos por su menor

Nexo Revista Científica / Vol. 30, No. 01, pp. 01-18 / Junio 2017 
productividad y tendencia a la formación de agregados que impiden que los rayos solares penetran con mayor profundidad;

- $\quad$ La superficie del agua está libre de toda materia sólida;

- Existe ausencia de plantas acuáticas y malas hierbas en los taludes.

\section{PROBLEMAS DE FUNCIONAMIENTO DE LAS LAGUNAS FACULTATIVAS}

En caso que la laguna facultativa esté presentando problemas en su funcionamiento, el Ing. Sergio Rolím (1999) afirma que se pueden presentar acumulación de materias flotantes, aparición de malos olores, desarrollo de coloraciones rosa o rojo, anomalías de flujo, crecimiento de malas hierbas y plantas acuáticas y desarrollo de mosquitos y otros insectos.

Por otra parte, las lagunas facultativas pueden sobrecargarse orgánicamente, en este caso, opera como laguna anaerobia. El fenómeno de sobrecarga en una laguna facultativa se refleja en la inhibición del desarrollo de las microalgas por la presencia del sulfuro de hidrógeno, ácidos volátiles o un ambiente fuertemente reductivo. La ausencia de luz debida al contenido de materia suspendida en el cuerpo de agua y la absorción de la radiación solar por las natas formadas (generalmente de color negro) fomentan la generación de sulfuro ferroso, limitando también la producción fotosinténtica de oxígeno. Una laguna operando en esas condiciones se encuentra propensa a un mal funcionamiento, generación de olores ofensivos, propagación de insectos y a una pobre eficiencia.

Al tomar en consideración que el Nitrógeno amoniacal se incrementa a través de procesos anaeróbicos, Yánez (1993) desarrolló una investigación en la que correlacionó la fracción de Nitrógeno Amoniacal versus la Cargas Superficial Aplicada en las Lagunas de San Juan en Perú. Como resultado de dicha investigación se determinó, que si la fracción de Nitrógeno Amoniacal del Afluente y el efluente de la laguna primaria es igual o mayor a la unidad, la laguna primaria se encuentra en proceso predominantemente anaerobio.

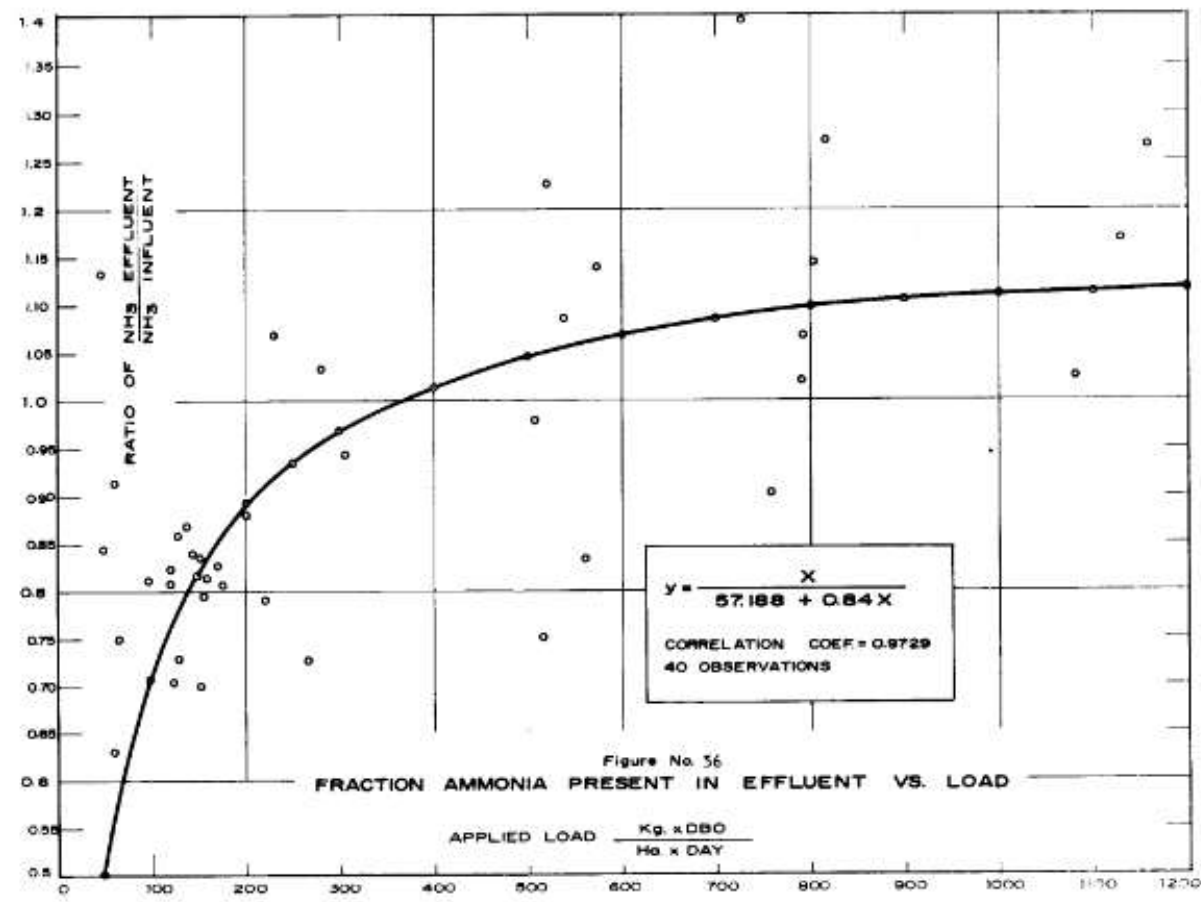

Figura 3. Relación de Nitrógeno Amoniacal vs Carga Superficial Aplicada Tomado de (Yanez F. , 1981, pág. 29)

Nexo Revista Científica / Vol. 30, No. 01, pp. 01-18 / Junio 2017 
De la figura 3 se obtiene la ecuación de correlación entre la fracción de Nitrógeno Amoniacal y la Carga Superficial Aplicada (CSA), válida únicamente para las lagunas de Perú y a una temperatura de $20^{\circ} \mathrm{C}$. Sin embargo, el criterio de relación de Nitrógeno Amoniacal como indicador de proceso predominante anaeróbico es válido y aplicable para lagunas primarias facultativas ubicadas en países con clima tropical.

\section{METODOLOGÍA}

Para seleccionar el método de cálculo de Carga Superficial Máxima, que permite un eficiente funcionamiento de remoción de materia orgánica y que a su vez optimiza área de terreno y recursos económicos aplicada al pacífico de Nicaragua se siguió la siguiente metodología.

\subsection{Selección del área de estudio}

Del total de 13 lagunas de estabilización en el pacífico de Nicaragua, se analizaron 2 plantas de tratamiento de aguas residuales, las cuales fueron "el patriarca" ubicada en el municipio de Nagarote y "San Rafael del sur" ubicada en el municipio del mismo nombre. De ambas plantas de tratamiento se estudió únicamente la laguna primaria facultativa, por ser la encargada de recibir la carga orgánica proveniente de las aguas residuales municipales.

La selección de dichas lagunas se rigió por los siguientes criterios:

- Ubicadas en alguno de los departamentos del Pacífico de Nicaragua.

- El sistema debe poseer Lagunas Primarias Facultativas.

- No debe mostrar síntomas visuales de condiciones anaeróbicas

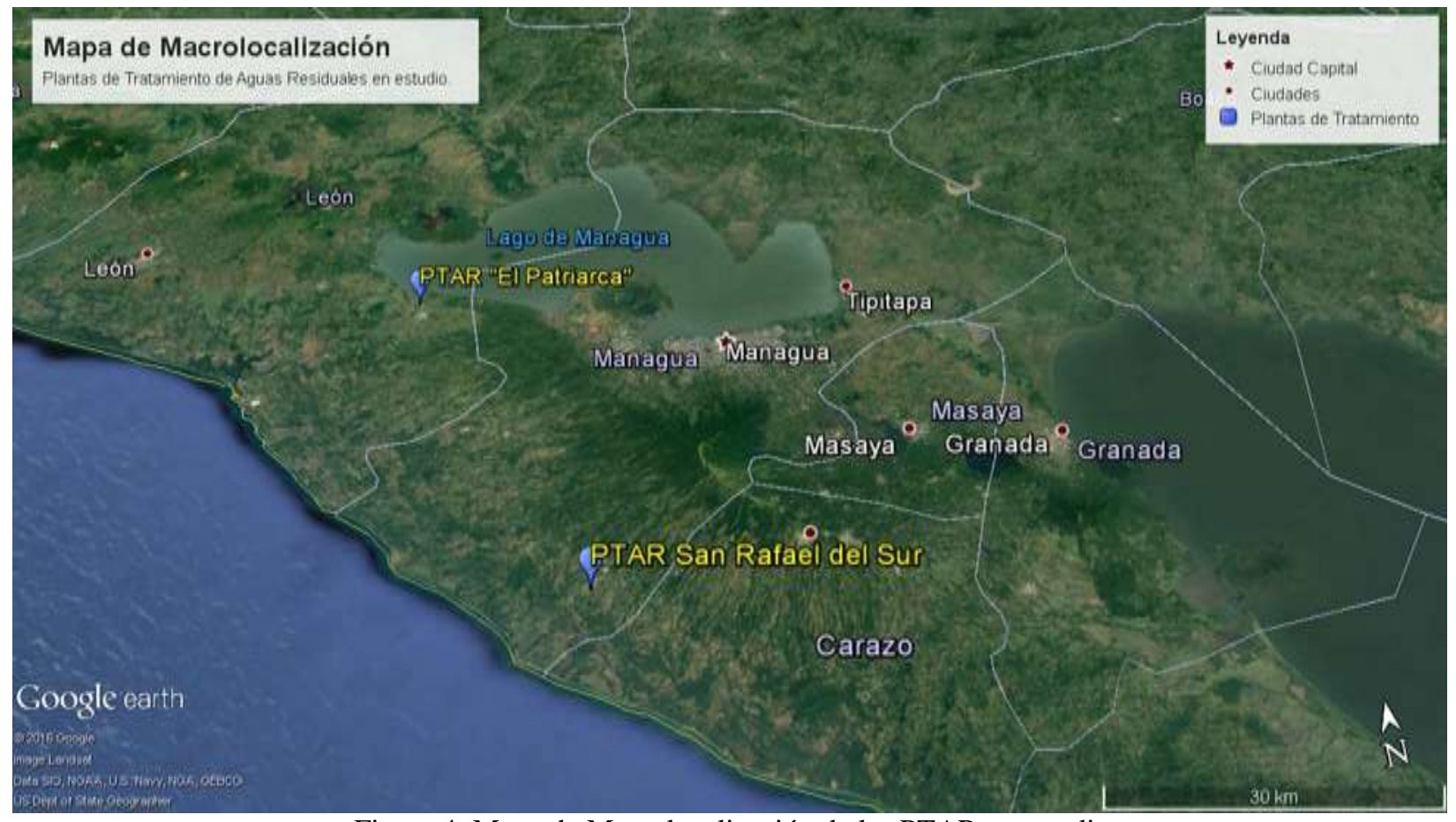

Figura 4. Mapa de Macrolocalización de las PTAR en estudio Fuente: Google earth

La PTAR "El Patriarca” está ubicada al suroeste de la ciudad Nagarote, departamento de León, consta de un solo módulo de lagunas de estabilización (primaria y secundaria). La laguna primaria tiene forma 
rectangular con un ancho de $69.2 \mathrm{~m}$ y largo $130.9 \mathrm{~m}$ para un área total de $9058.28 \mathrm{~m} 2 \mathrm{con}$ capacidad para tratar un caudal de diseño de 24.14 lps beneficiando a un total de 23000 habitantes.

La PTAR de San Rafael del sur está localizada en la zona sur del municipio de San Rafael del sur, departamento de Managua, cuenta dos módulos de lagunas de estabilización (primarias y secundarias) colocadas en paralelo. Sin embargo, tanto el pretratamiento como el módulo oeste fueron construidos recientemente. La laguna primaria tiene forma rectangular y tiene un área con un ancho de $40 \mathrm{~m}$ y largo de $80 \mathrm{~m}$ para un área total de $3200 \mathrm{~m} 2$ para tratar un caudal 5.4 lps, beneficiando un total de 2361 habitantes.

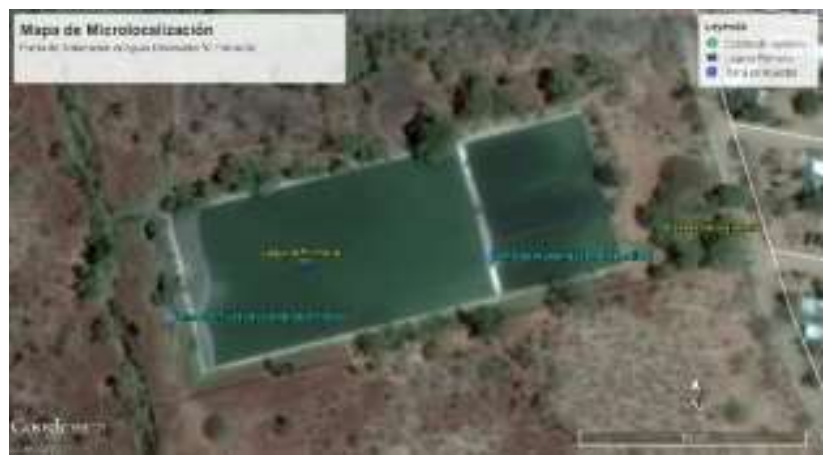

(a)

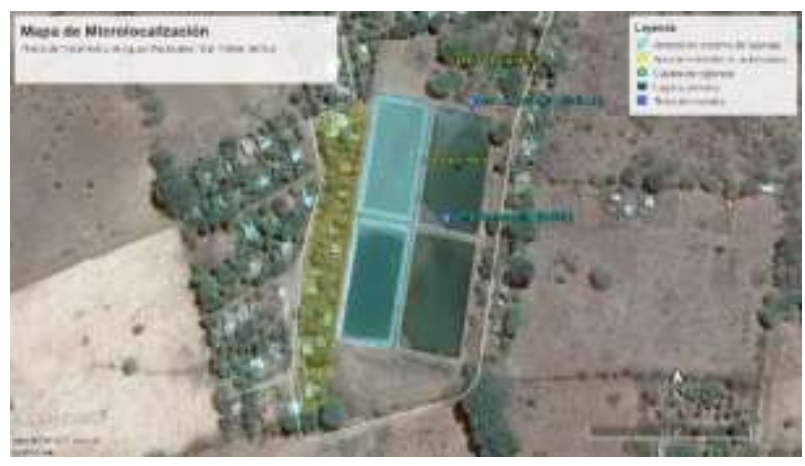

(b)

Figura 5. Mapa de microlocalización. (a) PTAR El Patriarca. (b) PTAR San Rafael del Sur Fuente: Google earth

\subsection{Caracterización físico-química del agua residual}

Para el desarrollo de esta investigación, se decidió realizar una serie de rondas de muestreos en las dos lagunas de estabilización seleccionadas. Se efectuaron un total de 4 rondas de muestreos para la laguna primaria "El Patriarca" y 3 rondas de muestreos para la laguna Primaria "San Rafael del Sur". En cada ronda de muestreo se recopilaron datos de campo (color del agua, $\mathrm{Ph}$ y temperatura del agua) y se recolectaron muestras compuestas de agua tanto en la entrada como en la salida de la laguna primaria. La temperatura ambiente se obtuvo

Cada muestreo compuesto duró 24 horas continuas y consistió en recolectar dos volumen de muestra de agua residual (en la entrada y la salida) en proporción al caudal de entrada de la PTAR (Gevara Vera \& León S., 1996). Para el cálculo del volumen de muestra se procedió con la siguiente ecuación:

$$
V m=\frac{3500 * Q_{i}}{24 * Q_{\text {prom }}}
$$

donde

Vm: Volumen de muestra $(\mathrm{ml})$

Qi: Caudal medido en cada hora (lps)

Qprom: Caudal promedio de la anterior ronda de muestreo (lps)

Las muestras recolectadas fueron transportadas al laboratorio para el análisis de la Demanda Bioquímica de Oxígeno $\left(\mathrm{DBO}_{5}\right)$ y Nitrógeno Amoniacal $\left(\mathrm{NH}_{4}\right)$. Se decidieron analizar únicamente estos parámetros porque nos interesaba la cantidad de materia orgánica biodegradable y el monitoreo de Nitrógeno Amoniacal para verificar si las lagunas continuaban trabajando en condiciones facultativas

En las dos PTAR seleccionadas no existía ningún mecanismo de medición de caudal. En el caso de la PTAR "El Patriarca" el agua residual ingresa por medio de un largo canal rectangular de concreto, en cambio, en la PTAR "San Rafael del Sur" el agua residual entra a la laguna por medio de un tubo de alcantarilla y un 
pequeño canal de distribución. Por consiguiente se procedió a medir el caudal de entrada con diferentes métodos para cada laguna.

En la PTAR "El Patriarca” se determinó una fórmula para el cálculo del caudal en función del tirante de agua dentro del canal rectangular. Dicha fórmula se generó como producto de un gráfico de dispersión de Caudal vs Tirante de agua (ver figura 6).

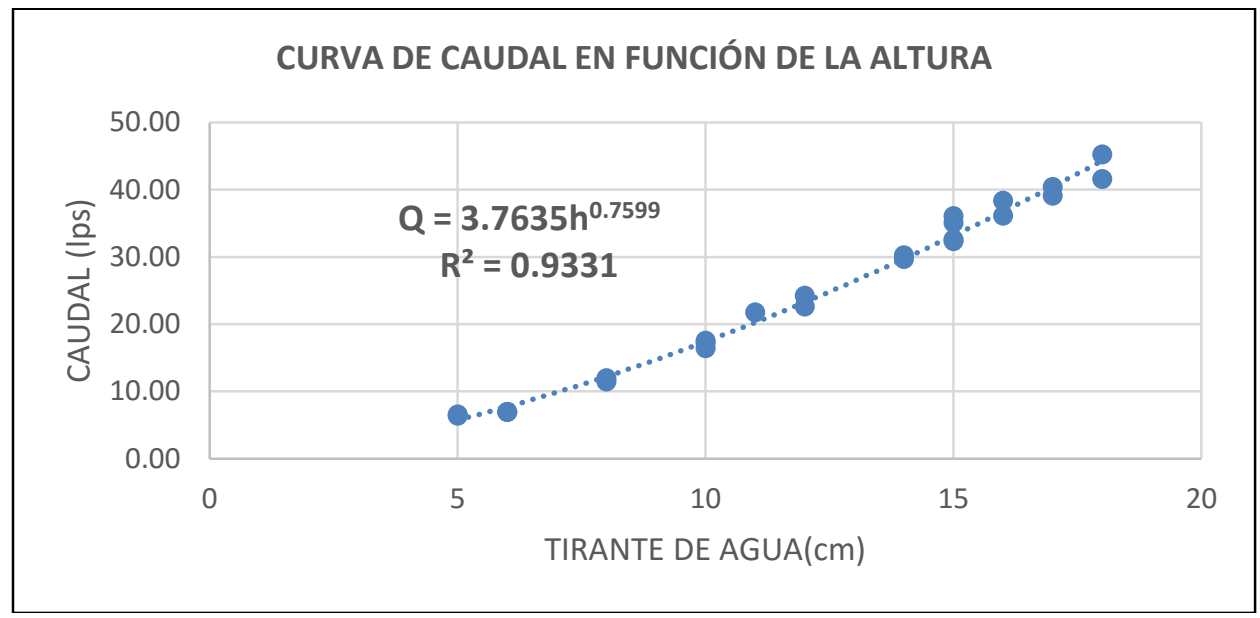

Figura 6. Curva de caudal en función del tirante de agua Fuente propia

Los datos que originaron el gráfico 18 fueron proporcionados por ENACAL, de acuerdo a sus mediciones históricas realizadas con el método de flotadores. De acuerdo al gráfico 18, el caudal de entrada de la PTAR "El Patriarca" está directamente relacionada al tirante de agua como se indica en la siguiente fórmula:

$$
Q=3.7635 * h^{0.7599}
$$

donde Q: Caudal de entrada a la laguna en lps.

h: tirante de agua en el canal de entrada de la laguna en $\mathrm{cm}$

En el caso de la PTAR "San Rafael del Sur" se determinó el caudal midiendo la velocidad de la corriente dentro del canal mediante un medidor de caudal digital (flow probe), el cual consiste en un bastón graduado con un molinete en el extremo inferior, que al girar con el paso del agua, mide la velocidad promedio del flujo y la muestra en la pantalla en el extremo superior.
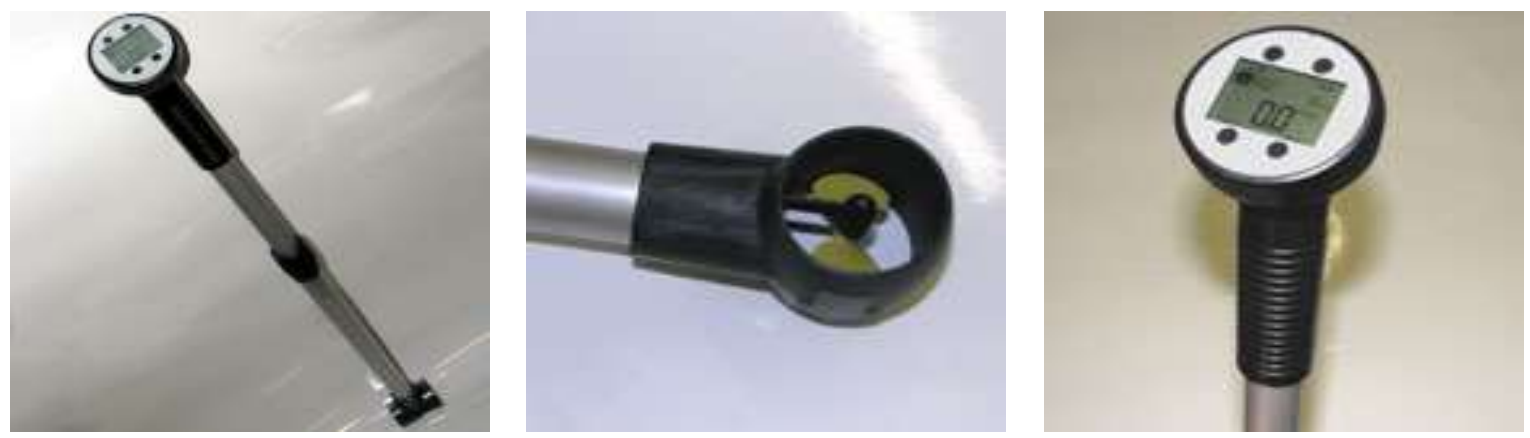

Figura 7. Medidor de flujo Global Water Flow Probe modelo FP211 Fuente http://www.globalw.com 


\subsection{Determinación de la Carga Superficial Aplicada (CSA) y porcentaje de Remoción}

Con los resultados de la $\mathrm{DBO}_{5}$ en la entrada de la laguna y su caudal promedio diario, se determinó la Carga Total Aplicada (fórmula 3). Al dividir la CTA entre el área superficial de la laguna primaria (ha) se obtuvo la Carga Superficial Aplicada (fórmula 4).

$$
\begin{gathered}
C T A=0,0864 * D B O_{5} * Q_{\text {mun }} \\
C S A=\frac{C T A}{A_{t}}
\end{gathered}
$$

Donde CSA: Carga Superficial Aplicada medida en KgDBO5/ha*d

CTA: Carga Total Aplicada medida en kgDBO5/d

Qmun: Caudal de aguas residuales a la entrada de la laguna primaria en lps.

Otros valores que se obtuvieron fueron la eficiencia de remoción de la laguna primaria y la carga orgánica promedio generada por persona diaria.

La eficiencia de remoción, expresada en porcentaje se obtuvo de restar la $\mathrm{DBO}_{5}$ a la entrada de la laguna con la $\mathrm{DBO}_{5}$ a la salida de la misma. Para ello se recurrió a la siguiente fórmula

$$
D B O_{r}=\frac{D B O_{E}-D B O_{S}}{D B O_{E}} * 100
$$

donde DBOr: Demanda Bioquímica de Oxígeno removido (\%)

$\mathrm{DBO}_{\mathrm{E}}$ : Demanda Bioquímica de Oxígeno en la entrada $(\mathrm{mg} / \mathrm{l})$

DBOs: Demanda Bioquímica de Oxígeno en la salida (mg/l)

La producción de carga orgánica per cápita se determinó dividiendo la Carga Total Aplicada entre el número de personas conectadas en el alcantarillado sanitario que conduce a la PTAR.

$$
D B O_{\text {percápita }}=\frac{C T A}{P} * 1000
$$

Donde $\mathrm{DBO}_{\text {percápita: }}$ Producción de carga orgánica por persona diaria $(\mathrm{gDBO} / \mathrm{hab} * \mathrm{~d})$

CTA: Carga total aplicada (kg DBO/d)

P: Población conectada al alcantarillado sanitario que conduce a la PTAR.

\subsection{Selección del método de CSM}

Para seleccionar el método de diseño de Carga Superficial Máxima, se calcularon cada una de las fórmulas propuestas en la tabla 1, utilizando los valores de latitud, radiación solar y temperatura ambiente y del agua para cada una de las lagunas en estudio. De acuerdo a los resultados obtenidos se aplicó el criterio de selección que consiste en que la Carga Superficial Máxima calculada debe ser siempre mayor que el valor real de Carga Superficial Aplicada. Será seleccionado el método que cumpla este criterio en ambas lagunas, en caso contrario el método genera lagunas con dimensiones poco eficientes. 


\section{ANÁLISIS E INTERPRETACIÓN DE RESULTADOS}

\subsection{Caracterización físico-química}

Color: Tanto en la laguna facultativa primaria de la PTAR "El Patriarca" como en la laguna "San Rafael del Sur" se observó claramente en el centro de la laguna un color verdoso, propio de cuerpos de agua con presencia de algas (ver figura 8). Desde esta perspectiva se deduce la existencia de oxígeno en la capa superior de las lagunas y por ende se garantiza la depuración de materia orgánica a través del proceso de oxidación aerobia. Es decir, guiados solamente por el color de la laguna

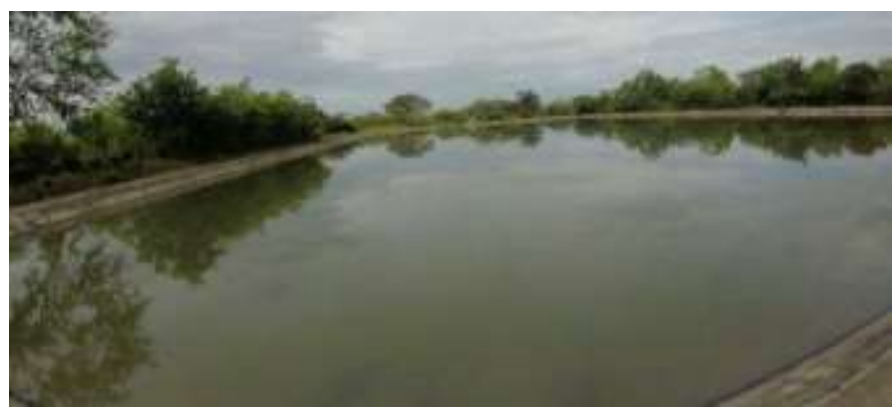

(a)

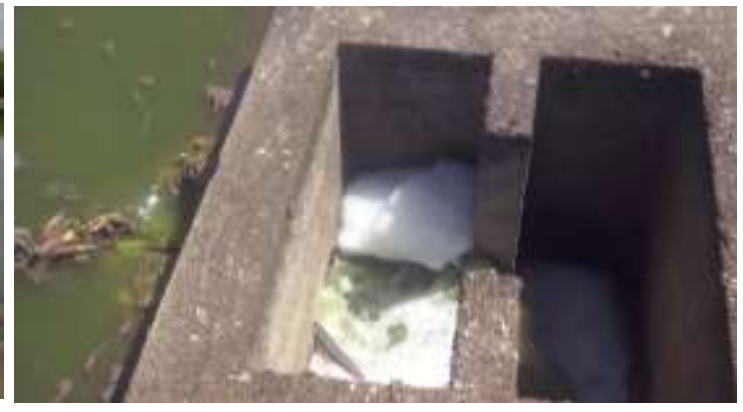

(b)

Figura 8. Presencia de algas en las lagunas primarias. (a) PTAR El Patriarca. (b) PTAR San Rafael del Sur Fuente: Propia

Caudal: En el siguiente gráfico se muestran los histogramas de caudales generados en los cuatro muestreos desarrollados en la PTAR "El Patriarca".
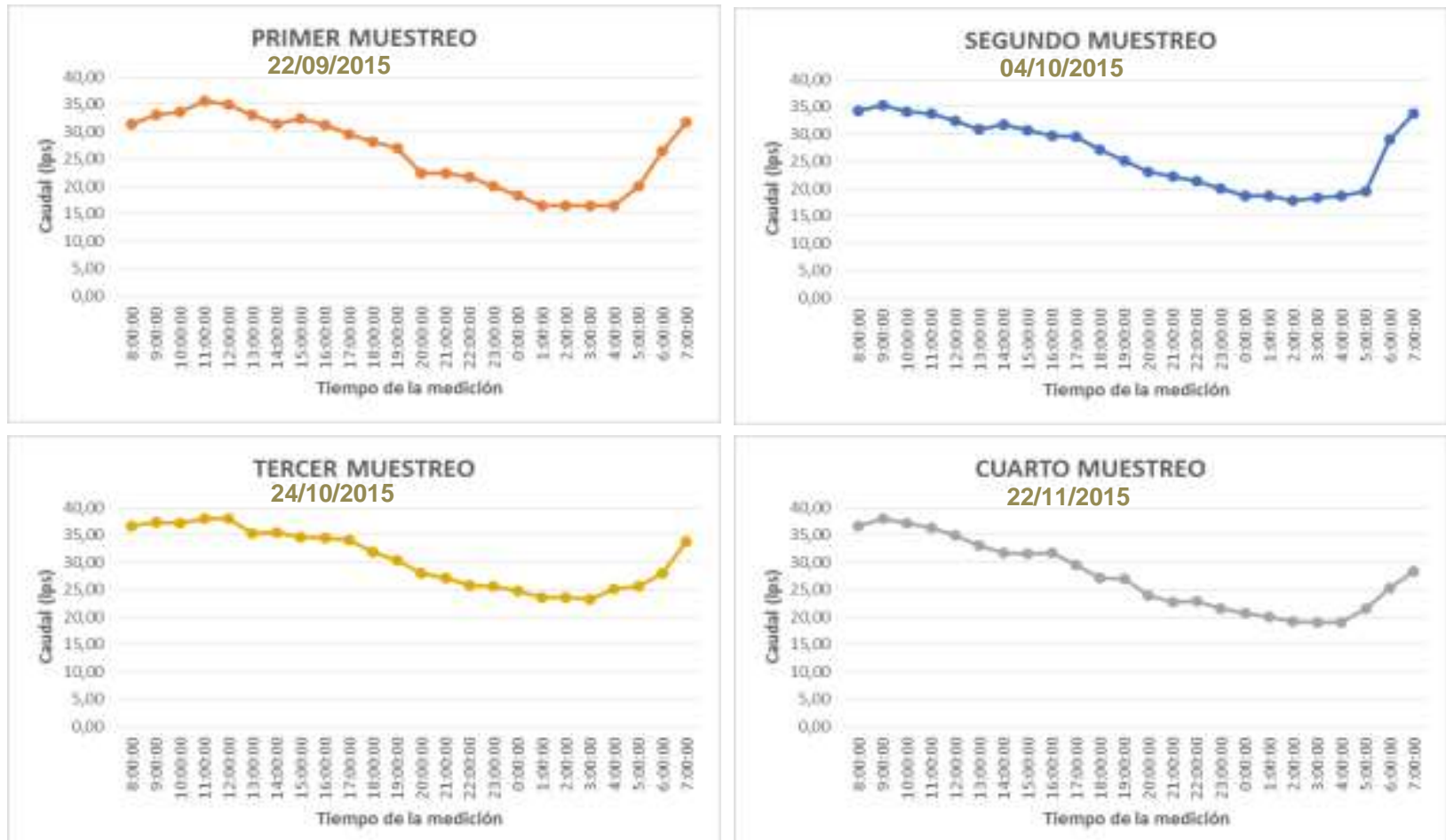

Figura 9. Histograma de caudales de la PTAR "El Patriarca" Fuente: Propia 
En la figura 9 se logra apreciar una similitud en la tendencia del histograma de caudales, generándose mayores caudales en los horarios de 7:00 am y 12:00 pm; y los menores caudales en horarios de la madrugada entre las 2:00 am-4:00am.

El caudal promedio registrado durante el período de muestreo fue de 27,76 lps, excediendo el caudal de diseño en un $15 \%$. Este exceso de caudal se debe probablemente a que la PTAR ha superado su período de diseño, por lo que el número de conexiones puede ser mayor que el proyectado.
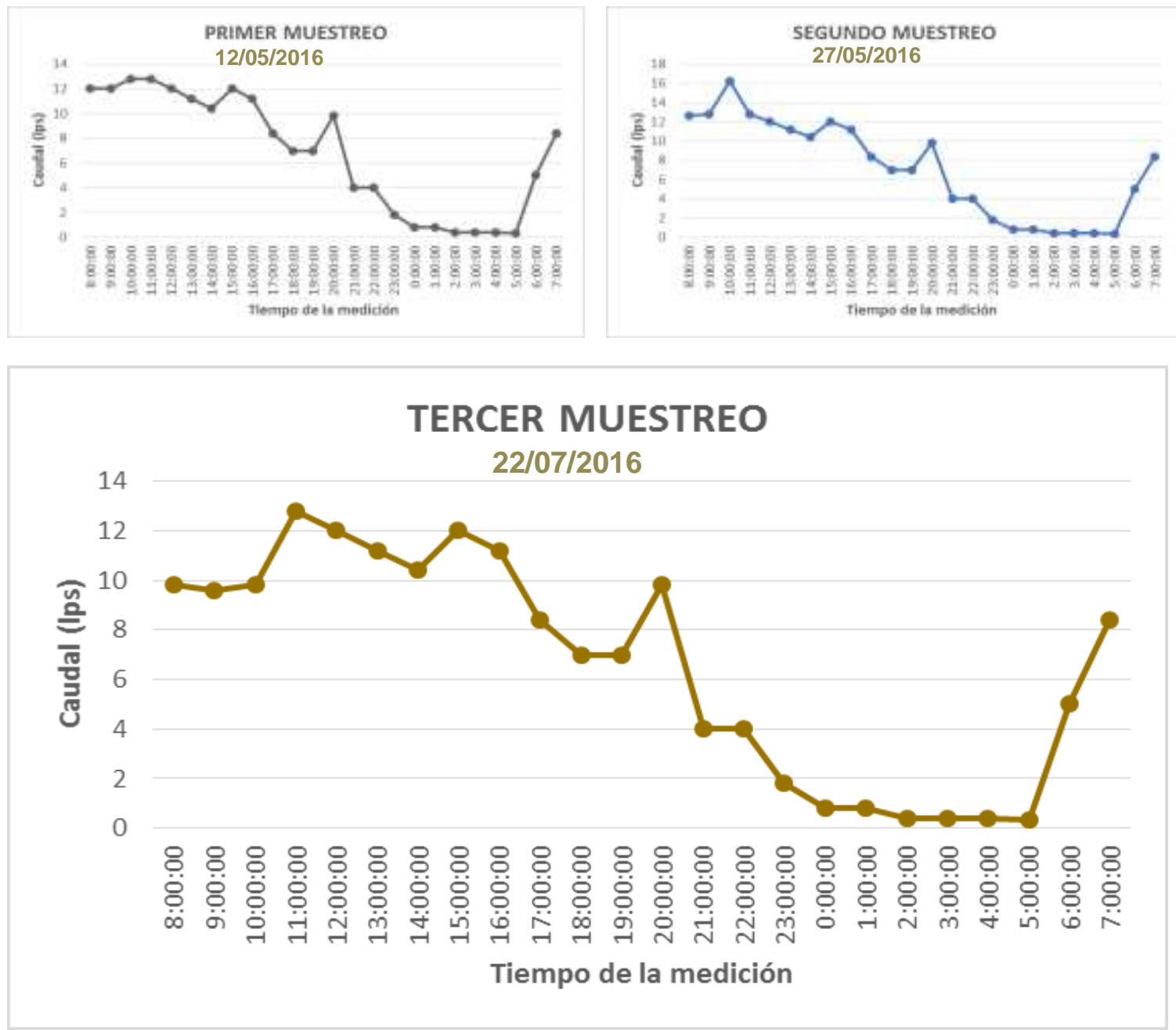

Figura 10. Histograma de caudales de la PTAR "San Rafael del Sur" Fuente: Propia

De acuerdo a los histogramas se aprecia que la PTAR "San Rafael del Sur" procesa mayores caudales durante la mañana, especialmente entre las 11:00 am-12:00 pm; y los menores caudales en horas de la madrugada entre las 2:00 am y las 4:00 am.

El caudal promedio registrado durante el período de muestreo es de 6,83 lps, superando el caudal de diseño en un $26,56 \%$. La diferencia de caudal obedece, de igual manera, a que probablemente la PTAR ha superado su vida útil y el número de conexiones al alcantarillado ha sido superado. 
Demanda Bioquímica de Oxígeno (DBO5): El municipio de Nagarote y San Rafael del Sur poseen por lo general conexiones domiciliares conectadas al alcantarillado sanitario, por lo tanto, se decidió medir la cantidad de materia orgánica mediante el análisis de $\mathrm{DBO}_{5}$. La figura 11 muestra los resultados de la $\mathrm{DBO}_{5}$ de los muestreos realizados tanto a la entrada como la salida de la laguna primaria de la PTAR "El Patriarca” y "San Rafael del Sur".

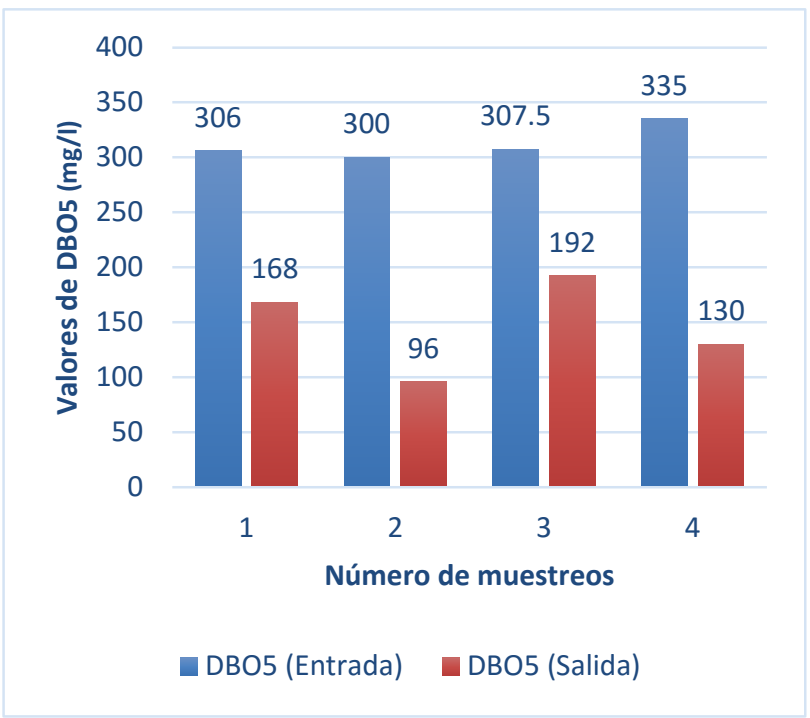

(a)

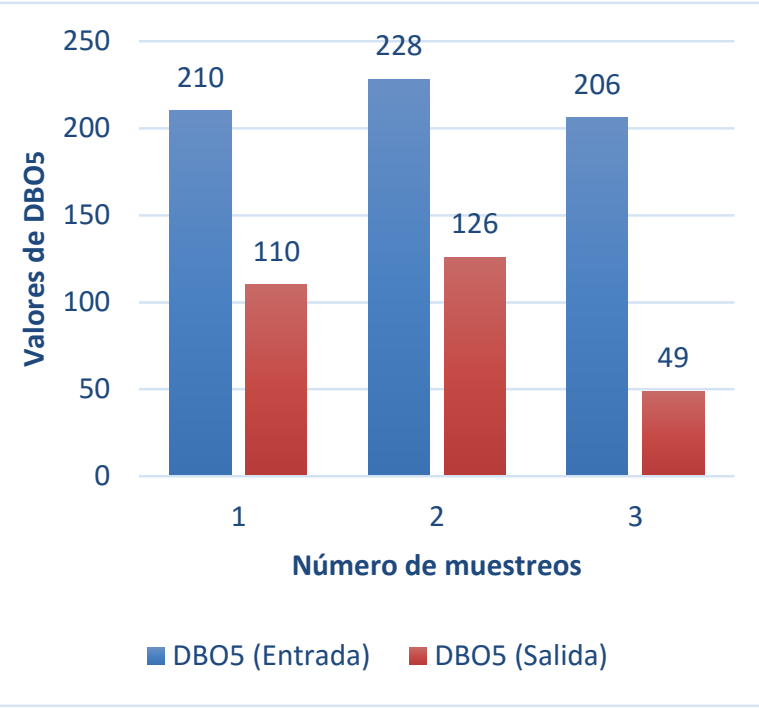

(b)

Figura 11. Resultados de $\mathrm{DBO}_{5}$. (a) PTAR “El Patriarca” (b) PTAR “San Rafael del Sur” Fuente: Propia

De acuerdo a los resultados mostrados, los valores de $\mathrm{DBO}_{5}$ en la salida resultaron menores que los valores de $\mathrm{DBO}_{5}$ en la entrada de la laguna primaria de ambas PTAR. Para determinar la eficiencia de remoción de las lagunas primarias en estudio se utilizó la fórmula 5. Los resultados se pueden apreciar en la tabla 2.

Tabla 2. Eficiencia de remoción de la laguna primaria de la PTAR "El Patriarca" y la PTAR "San Rafael del Sur"

\begin{tabular}{ccccc|cccc}
\hline No & Fecha & $\begin{array}{c}\text { DBO5 Ent. } \\
(\mathbf{m g} / \mathbf{l})\end{array}$ & $\begin{array}{c}\text { DBO5 Sal. } \\
(\mathbf{m g} / \mathbf{l})\end{array}$ & $\mathbf{\%}$ Rem. & Fecha & $\begin{array}{c}\text { DBO5 Ent. } \\
(\mathbf{m g} / \mathbf{l})\end{array}$ & $\begin{array}{c}\text { DBO5 Sal. } \\
(\mathbf{m g} / \mathbf{l})\end{array}$ & \% Rem. \\
\hline $\mathbf{1}$ & $22 / 09 / 2015$ & 306 & 168 & $45 \%$ & $12 / 05 / 2016$ & 210 & 110 & $48 \%$ \\
$\mathbf{2}$ & $04 / 10 / 2015$ & 300 & 96 & $68 \%$ & $27 / 05 / 2016$ & 228 & 126 & $45 \%$ \\
$\mathbf{3}$ & $24 / 10 / 2015$ & 307,5 & 192 & $38 \%$ & $22 / 07 / 2016$ & 206 & 49 & $76 \%$ \\
$\mathbf{4}$ & $22 / 11 / 2015$ & 335 & 130 & $61 \%$ & --- & --- & --- & -- \\
\hline \multicolumn{2}{r}{ Promedio } & 312,13 & 146,50 & $53 \%$ & & 214,67 & 95,00 & $56 \%$ \\
\hline \multicolumn{7}{r}{ Fuente propia }
\end{tabular}

La tabla 2 muestra que la laguna primaria de la PTAR "El Patriarca presenta un porcentaje de remoción de un 53\% de DBO5, y la PTAR "San Rafael del Sur" un porcentaje de remoción del 56\%. Para ambos casos, se considera que las lagunas primaras funcionan con un buen porcentaje de remoción, debido a que dicho valor es cercano al rango de 70-85\% de DBO5 establecido en la NTON 05-027-05 (MARENA, 2006) para todo el sistema de tratamiento conformado por lagunas facultativas. Además, el valor promedio de $\mathrm{DBO}_{5}$ a la salida de la laguna primaria de la PTAR "San Rafael del Sur" es $95 \mathrm{mg} / \mathrm{l}$, que resulta siendo menor a $110 \mathrm{mg} / \mathrm{l}$, valor máximo establecido en el decreto 33-95 (INAA, 1995). 
Nitrógeno Amoniacal: Las siguientes tablas y figuras muestran los resultados obtenidos de los análisis de Nitrógeno Amoniacal $\left(\mathrm{NH}_{3}\right)$ realizados durante el período de muestreo, tanto en la entrada como en la salida de las lagunas primarias de las PTAR en estudio.

Tabla 3. Valores de Nitrógeno Amoniacal (NH3) en la PTAR "El Patriarca"

\begin{tabular}{ccccccccc}
\hline Muestreo & \multirow{2}{*}{ Fecha } & \multirow{2}{*}{ NH3 Ent. } & \multirow{2}{*}{ NH3 Sal. } & \multirow{2}{*}{ Fracción NH3 } & Fecha & $\begin{array}{c}\text { NH3 } \\
\text { Ent. }\end{array}$ & $\begin{array}{c}\text { NH3 } \\
\text { Sal. }\end{array}$ & $\begin{array}{c}\text { Fracción } \\
\text { NH3 }\end{array}$ \\
\hline $\mathbf{1}$ & $22 / 09 / 2015$ & 14 & 23,9 & 1,71 & $12 / 05 / 2016$ & 16,68 & 9,93 & 0,60 \\
$\mathbf{2}$ & $04 / 10 / 2015$ & 31,61 & 16,46 & 0,52 & $27 / 05 / 2016$ & 17,48 & 14,31 & 0,82 \\
$\mathbf{3}$ & $24 / 10 / 2015$ & 20,82 & 17,35 & 0,83 & $22 / 07 / 2016$ & 18,45 & 15,22 & 0,82 \\
$\mathbf{4}$ & $22 / 11 / 2015$ & 21,5 & 9,63 & 0,45 & --- & --- & -- & -- \\
\hline
\end{tabular}

Fuente propia

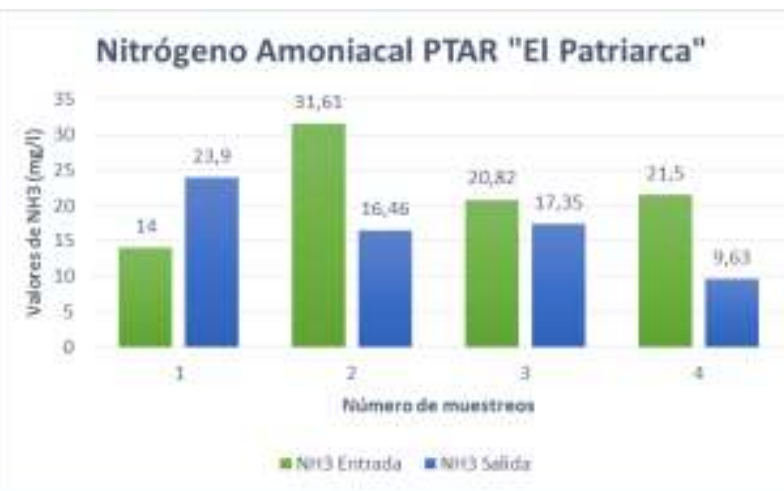

(a)

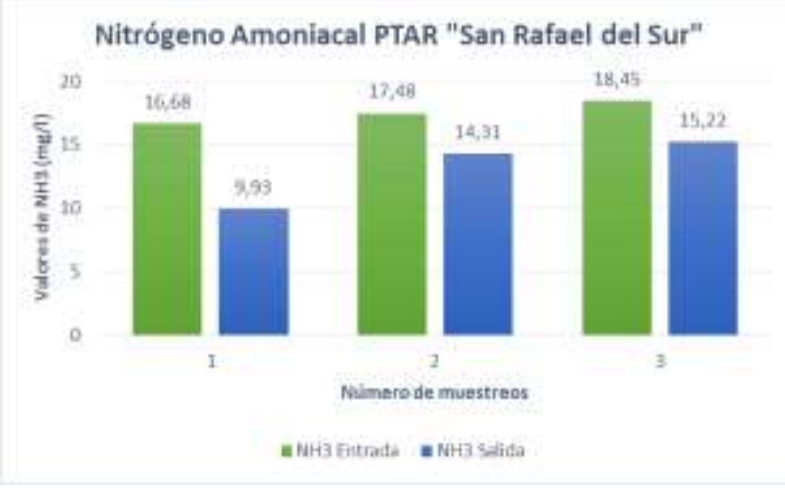

(b)

Figura 12. Resultados de $\mathrm{NH}_{3}$. (a) PTAR "El Patriarca". (b) PTAR "San Rafael del Sur" Fuente: Propia

De acuerdo a los resultados obtenidos, tanto la laguna primaria de la PTAR "El Patriarca", como la PTAR "San Rafael del Sur" remueven Nitrógeno Amoniacal en la mayoría de los casos. Al hacer una división entre el $\mathrm{NH}_{3}$ a la salida y el $\mathrm{NH}_{3}$ de la entrada, se verifica que en la mayoría de los muestreos se obtienen valores menores a 1. Por tanto, se puede deducir que de acuerdo a la fracción de $\mathrm{NH}_{3}$, la laguna Primaria $\mathrm{El}$ Patriarca y San Rafael del Sur, presentan un proceso predominantemente facultativo.

\subsection{Carga Superficial Aplicada}

Para calcular la Carga Total Aplicada (CTA) se utilizó la fórmula 3 descrita en la metodología. Del mismo modo, la Carga Superficial Aplicada en la laguna primaria de la PTAR "El Patriarca" se calculó por medio de la fórmula 4, tomando que el área superficial de la laguna es de $9058.28 \mathrm{~m} 2$.

Tabla 4. Carga Superficial Aplicada en la PTAR "E1 Patriarca"

\begin{tabular}{cccccc}
\hline No & Fecha & $\begin{array}{c}\text { Caudal Prom. } \\
(\mathbf{l p s})\end{array}$ & $\begin{array}{c}\text { DBO5 Entrada } \\
(\mathbf{m g} / \mathbf{l})\end{array}$ & $\begin{array}{c}\text { CTA } \\
\text { kg DBO/d }\end{array}$ & $\begin{array}{c}\text { CSA } \\
\text { kg DBO/(Ha*d) }\end{array}$ \\
\hline $\mathbf{1}$ & $22 / 09 / 2015$ & 26,29 & 306 & 695,02 & 767,27 \\
$\mathbf{2}$ & $04 / 10 / 2015$ & 26,50 & 300 & 686,98 & 758,40 \\
$\mathbf{3}$ & $24 / 10 / 2015$ & 30,77 & 307,5 & 817,38 & 902,36 \\
$\mathbf{4}$ & $22 / 11 / 2015$ & 27,47 & 335 & 795,20 & 877,87 \\
\multicolumn{2}{c}{ Promedio } & 27,76 & 312,13 & 748,65 & 826,48 \\
\hline
\end{tabular}

Fuente propia 
De acuerdo a los resultados de la tabla 4, la laguna primaria "El Patriarca" es capaz de recibir una CSA promedio de $826,48 \mathrm{kgDBO} / \mathrm{ha} * \mathrm{~d}$ y trabajar con procesos predominantemente facultativos. Esto quiere decir que la laguna primaría todavía no alcanza los valores máximos de Carga Superficial y funciona correctamente con la carga superficial que actualmente posee.

Por otra parte, haciendo uso de la ecuación 6, se calculó el aporte per cápita de carga orgánica. En el caso de la ciudad de Nagarote, la población conectada al alcantarillado es de 14466 habitantes (PROATAS, 2013). El resultado fue el siguiente:

$$
\begin{gathered}
D B O_{\text {percápita }}=\frac{C T A}{P} * 1000 \\
D B O_{\text {percápita }}=\frac{748,65}{14466} * 1000=51,75 \mathrm{gDBO5} /(\text { hab } * d)
\end{gathered}
$$

El aporte per cápita para la PTAR "El Patriarca” es de 51,75 gDBO5/hab*d. Dicho resultado es bastante cercano a los 54 gDBO5/hab*d (Tercero Talavera, 2015).

En el caso de la PTAR "San Rafael del Sur" se calculó la Carga Total Aplicada y la Carga Superficial aplicada, considerando que el área superficial de la laguna primaria es de 0.28 hectáreas.

Tabla 5. Carga Superficial Aplicada en la PTAR "San Rafael del Sur"

\begin{tabular}{cccccc}
\hline No & Fecha & $\begin{array}{c}\text { Caudal Prom. } \\
\text { (lps) }\end{array}$ & $\begin{array}{c}\text { DBO5 Entrada } \\
\text { (mg/l) }\end{array}$ & $\begin{array}{c}\text { CTA } \\
\text { kg DBO/d }\end{array}$ & $\begin{array}{c}\text { CSA } \\
\text { kg DBO/(Ha*d) }\end{array}$ \\
\hline $\mathbf{1}$ & $12 / 05 / 2016$ & 6,87 & 210 & 124,71 & 445,39 \\
$\mathbf{2}$ & $27 / 05 / 2016$ & 9,59 & 228 & 188,95 & 674,81 \\
$\mathbf{3}$ & $22 / 07 / 2016$ & 8,01 & 206 & 143,57 & 569,74 \\
\multicolumn{2}{c}{ Promedio } & 8,16 & 214,67 & 152,41 & 544,32 \\
\hline \multicolumn{2}{l}{ Fuente propia }
\end{tabular}

De acuerdo a los resultados de la tabla 5, la laguna primaria "San Rafael del Sur" es capaz de recibir una CSA promedio de $544,32 \mathrm{kgDBO} / \mathrm{ha} * \mathrm{~d}$ y trabajar con procesos predominantemente facultativos. Esto quiere decir que la laguna primaría todavía no alcanza los valores máximos de Carga Superficial y funciona correctamente con la carga superficial que actualmente posee.

Por otro lado, la población conectada al alcantarillado sanitario de la ciudad de San Rafael del Sur es de 2361 habitantes (PROATAS, 2013), por lo que el aporte percápita fue el siguiente:

$$
D B O_{\text {percápita }}=\frac{152,41}{2361} * 1000=64,55 \mathrm{gDBO} 5 /(\mathrm{hab} * d)
$$

El aporte per cápita para la PTAR “San Rafael del Sur” es de 64,55 gr DBO5/hab*d. Dicho valor es mayor al valor per cápita teórico para el diseño de lagunas de estabilización. (Tercero Talavera, 2015).

\subsection{Carga Superficial Máxima}

Se procedió a calcular la Carga Superficial Máxima en cada laguna en estudio, por medio de los diferentes métodos propuestas en la tabla 1. 
Tabla 6. Resumen de los cálculos de Carga Superficial Máxima PTAR "El Patriarca"

\begin{tabular}{|c|c|c|c|c|c|c|c|c|c|c|}
\hline \multirow{2}{*}{ No } & \multirow{2}{*}{ Nombre de is formula } & \multirow{2}{*}{ Fórmula } & \multirow{2}{*}{\multicolumn{2}{|c|}{ Datos }} & \multirow{2}{*}{ CाA } & \multicolumn{5}{|c|}{ Resultado } \\
\hline & & & & & & CSM & Area req. $(\mathrm{Ha})$ & Excese (ha) & x. & $\operatorname{cs} A$ \\
\hline \multirow{2}{*}{1} & Ascevala $(1970)$ & $2 \mathrm{~S}=375-6.25 \mathrm{~L}$ & Latisud & 12,281 & \multirow{9}{*}{748,646} & 292,78 & 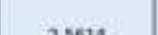 & save & 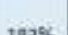 & \multirow{9}{*}{826,490} \\
\hline & Corrección por elev. & {$\left[1+\left(3 \cdot 10^{-4}\right) E\right]$} & Elevación (msnm) & 68,000 & & 1,02 & 20014 & 1,0000 & $103 \pi$ & \\
\hline 2 & Ritmann \& MeCarty (2001) & $C S M=69.75 \cdot R S$ & Rs $\left[\mathrm{kWh} / \mathrm{m} \mathbf{2}^{*} \mathrm{~d}\right)$ ] = & 5,29 & & 368,98 & 2,0290 & 1,1232 & 1246 & \\
\hline 3 & Mc Grary \& Pescod (1970) & $2 \mathrm{~S}=60.26(1.0993)^{\mathrm{Tu}}$ & T.amb("C) & 27,2 & & 790,49 & $0,94 \pi$ & 0,0413 & 25. & \\
\hline 4 & Mara (1987) & $\lambda 5=350(1107-0.0027)^{t+3}$ & t.amb $\left({ }^{\circ} \mathrm{C}\right)$ & 27,2 & & 391,78 & 1,9109 & 1,0051 & 1113 & \\
\hline 5. & Artar (1983) & $\lambda S=20 T-60$ & T.amb $\left({ }^{\circ} \mathrm{C}\right)$ & 27,2 & & 434,00 & 1,5468 & 0,6410 & $n \mathrm{~s}$ & \\
\hline 6 & México $(2007)$ & $\lambda s=250(1.085)^{r-2 \pi}$ & T.. amb (c) $c$ ) & 27,2 & & 449,82 & 1.6643 & 0,7585 & 846 & \\
\hline 7 & Nomma 05.090 Perí (2006) & $C_{d}=250(1.05)^{7-20}$ & Tagua $\left[{ }^{*} \mathrm{C}\right\rangle$ & $30, \pi$ & & 422,81 & $1, \pi 706$ & 0,8643 & $95 \%$ & \\
\hline 8 & Yainer, 1993 & $L_{1}=357.4 \cdot 1.085^{T .20}$ & Tagua $\left[{ }^{\circ} \mathrm{C}\right]$ & 30.77 & & $86 Q 46$ & 0,8700 & $-0,0358$ & $-4 \%$ & \\
\hline
\end{tabular}

Tabla 7. Resumen de los cálculos de Carga Superficial Máxima PTAR “San Rafael del Sur”

\begin{tabular}{|c|c|c|c|c|c|c|c|c|c|c|}
\hline \multirow{2}{*}{ No } & \multirow{2}{*}{ Nombre do la formula } & \multirow{2}{*}{ Fórmula } & \multirow{2}{*}{\multicolumn{2}{|c|}{ Datos }} & \multirow{2}{*}{ CTA } & \multicolumn{5}{|c|}{ Resultado } \\
\hline & & & & & & $\operatorname{csM}$ & Ares ren: (Ha) & Excess (ha) & $\mathbf{W}$ & C5A \\
\hline \multirow{2}{*}{1} & \multirow{2}{*}{$\begin{array}{c}\text { Arceivala }(1970) \\
\text { Correccico por elevación }\end{array}$} & $25-375-6251$ & Latitud & 11,830 & \multirow{9}{*}{152,410} & 291,11 & & & & \multirow{9}{*}{544,320} \\
\hline & & {$\left[1+\left(3 \times 10^{-4}\right) E\right]$} & Elevación (msnm) & 134 & & 1,03 & 0,5236 & 0,2036 & 645 & \\
\hline 2 & Rirtmann \& MoCarty (2001) & $C S M=69.75 \cdot R S$ & RS $($ KWW/m/2*d $)=$ & 6,32 & & 440,47 & 0,3460 & 0,0260 & $8 \%$ & \\
\hline 3 & Me Gary \& Pescod (1970) & $\lambda S=60.26(1.0993)^{\mathrm{Ta}}$ & T.anb ( $(2)$ & 26.9 & & $768, A 1$ & 0,1983 & $-0,1217$ & $-38 \%$ & \\
\hline 4 & Mara (1987) & $\lambda S=350(1.107-0.002 \tau)^{t-11}$ & T.anb $\left({ }^{*} \mathrm{C}\right)$ & 269 & & 365,22 & 0,3946 & 0,0746 & $23 \mathrm{~N}$ & \\
\hline 5 & Anthar (1983) & $\lambda \mathrm{s}=20 \mathrm{~T}-60$ & T.Amb $\left({ }^{*} \mathrm{C}\right)$ & 26.9 & & 478,00 & 0,3188 & $-0,0012$ & os & \\
\hline 6 & México (2007) & $\lambda s=250(1.085)^{t-20}$ & T.amb $\left.f^{*} \mathrm{C}\right)$ & 269 & & 438,94 & 0,3472 & 0.0272 & $9 \%$ & \\
\hline 7 & Norma OS.090 Perí (2006) & $C_{A}=250(1.05)^{7-30}$ & Tagus $(* \mathrm{C}$ & 29,65 & & 400,33 & 0,3807 & 0,0607 & $19 \%$ & \\
\hline 8 & Yänez, 1993 & $L_{1}=357.4 * 1.085^{T-20}$ & Tagua $\langle\circ C$ & 29.65 & & 785,33 & 0,1941 & $-0,1259$ & $-39 \%$ & \\
\hline
\end{tabular}

Fuente propia

De acuerdo a los resultados obtenidos al calcular la Carga Superficial Máxima con todos los métodos disponibles, se define que el método de Carga Superficial Máxima que mejor se adecua a las dos lagunas de estabilización en estudio es la propuesta por CEPIS a través de Fabián Yánez, debido a que genera una CSM mayor que la CSA y el cálculo del área da un valor muy aproximado al área real de las lagunas primarias. El resto de métodos da como resultados CSM menores a la CSA de las lagunas en estudio y por consiguiente área de laguna mayor al área que poseen actualmente.

\section{CONCLUSIONES}

1. De acuerdo a las observaciones realizadas en las lagunas primarias de las PTAR seleccionadas se concluye que ambas lagunas presentan en lo general un proceso predominantemente facultativo, debido a: la presencia de algas, la remoción de más del $50 \%$ de la materia orgánica y presenta una correlación de Nitrógeno Amoniacal entre la salida y la entrada con un valor menor que la unidad. Por otro lado, las PTAR "El Patriarca" y "San Rafael del Sur" presentan un exceso de caudal de entrada de aguas residuales de 15\% y $26,56 \%$ respectivamente, en relación a su caudal de diseño.

2. Los análisis de laboratorio de DBO5 en la entrada y la salida de las PTAR en estudio, demuestran que la PTAR "El Patriarca" recibe una Carga Superficial Aplicada de 826,48 kg de DBO5/ha*d, produciendo cada poblador un aporte per cápita de 51,75 gr DBO5/hab*d. Para la PTAR "San Rafael del Sur" Carga Superficial Aplicada recibida en su laguna primaria es de 544,32 kg de DBO5/ha*d asignando a cada habitante un aporte per cápita de 64,55 gr DBO5/hab*d.

3. La mayoría de métodos producen valores de Carga Superficial Máxima muy conservadores y áreas de lagunas sobredimensionadas. En el caso de la PTAR "El Patriarca", se determinó un rango de CSM de 
292,28 - 860,46 KgDBO5/ha*d y un rango de áreas de laguna de 2,56-0,87 hectáreas. En el caso de la laguna de estabilización "San Rafael del Sur" se determinó un rango de CSM de 291, 11-785,33 $\mathrm{KgDBO} / \mathrm{ha}$ *d y un rango de área de 0,52-0,19 ha.

4. El método de Carga Superficial Máxima que mejor se adecua al diseño de lagunas de estabilización en estudio, es la propuesta por el CEPIS a través de Fabián Yánez, ya que logra determinar una CSM mayor que la CSA para ambas lagunas estudiadas. El resto de métodos producen valores de CSM muy conservadores y áreas de lagunas sobredimensionadas, a excepción del método de Mc Garry and Pescod, que brinda un valor de CSM aproximado al de Yánez.

\section{AGRADECIMIENTOS}

Este trabajo fue posible gracias al patrocinio del Consejo Nicaragüense de Ciencia y Tecnología (CONICYT) a través de los Fondos Concursables de Estímulo a la Investigación e Innovación Tecnológica (FOCEIT).

\section{REFERENCIAS}

Arthur, J. (1983). Notes on the Design and Operation of Waste Stablilization Ponds in Warm Climates of Developing Countries. World Bank Technical Paper.

Comisión Nacional del Agua. (2007). Diseño de Lagunas de estabilización. Manual de Agua Potable, Alcantarillado y Saneamiento. D.F., México: Secretaría de Medio Ambiente y Recursos Naturales.

Cubillos Z, A. (Diciembre de 2001). Estado del arte en el diseño de lagunas de estabilización. Ingeniería y Competitividad, 3(2), 80-91.

Gevara Vera, A., \& León S., G. (1996). Propuesta metodológica Evaluacion de lagunas de estabilización. Lima: Centro Panamericano de Ingeniería Sanitaria y Ciencias del Ambiente.

INAA. (26 de 06 de 1995). Disposiciones para el control de la contaminación provenientes de descargas de aguas residuales domésticas, industriales y agropecuarias. La Gaceta Diario oficial, págs. 2186-2210.

INAA. (2004). Guía técnica para el diseño de alcantarillado sanitario y sistemas de tratamiento de aguas residuales. Managua: Instituto Nicaraguense de Acueductos y Alcantarillado.

Mara, D. D. (1997). Design Manual for Waste Stabilization Ponds in India. Leeds: Lagoon Technology International Ltd.

Mara, D. D. (2004). Domestic Wastewater Treatment in Developing Countries. London, UK: Earthscan.

MARENA. (10 de mayo de 2006). NTON 05-027-05 Norma Técnica Obligatoria para regular los sistemas de tratamiento de aguas residuales y su reuso (NTON 05027-05). La Gaceta, Diario oficial(90), págs. 35193531. Obtenido de http://legislacion.asamblea.gob.ni/Normaweb.nsf/xpNorma.xsp?documentId=3B3583B8C7D4EE3206257 9BC007B7023\&action=openDocument

Ministerio de Transporte, Comunicaciones, Vivienda y Construcción. (23 de Mayo de 2006). Norma OS.090. El Peruano, págs. 84-104. 
Carlos M. Vanegas-Benavides y Rosa Verónica Reyes Rodríguez

Platzer, M. (2002). Investigaciones y experiencias con filtros biológicos en Nicaragua, Centroamérica. XXVIII Congreso Interamericano de Ingeniería Sanitaria y Ambiental. Cancún: AIDIS.

PROATAS. (2013). Diagnóstico general de 35 Sistemas de tratamiento de aguas residuales de ENACAL. Managua: Programa de Asistencia Técnica en Agua y Sanemamiento, GIZ.

PROATAS. (2013). Diagnóstico General de 35 Sistemas de Tratamiento de Aguas Residuales de ENACAL. Programa de Asistencia Técnica en Agua y Saneamiento, Managua.

Rittmann, B., \& McCarty, P. (2001). Environmental Biotechnology: Principles and Applications. New York: McGraw-Hill Book Co.

Rolim, S. (1999). Lagunas de estabilización ¿Por qué no usarlas? Seminario Internacional Tratamiento de aguas residuales a través de humedales naturales y artificiales y lagunas de estabilización. Bogotá: Organización Panamericana de la Salud.

Tercero Talavera, S. (2015). Caracterización del agua residual urbana de Nicaragua en términos de la Demanda Bioquímica de Oxígeno, $\mathrm{DBO5}-20^{\circ} \mathrm{C}$. Managua.

Von Sperling, M. (2007). Wastewater, Characteristics, treatment and disposal. Biological Wastewater treatment series (Vol. 1). London: IWA Publishing.

Yanez, F. (1981). Avances en el tratamiento de aguas residuales por lagunas de estabilización. documentos técnicos, 7. Lima: Centro Panamericano de Ingeniería Sanitaria y Ciencias del Ambiente.

Yánez, F. (1984). Reducción de organismos patógenos y diseño de lagunas de estabilización. Santiago: XIX Congreso Interamericano de Ingeniería Sanitaria y Ambiental (AIDIS).

Yanez, F. (1993). Lagunas de Estabilización, Teoría, Diseño, Evaluación y Mantenimiento. Cuenca: Empresa Pública Municipal de Teléfonos, Agua Potable y Alcantarillado.

\section{SEMBLANZA DE LOS AUTORES}

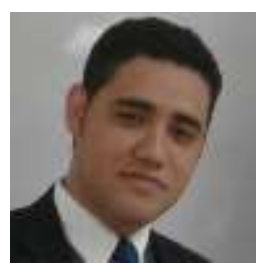

Carlos M. Vanegas-Benavides: Obtuvo el grado de Ingeniero Civil en la Universidad Nacional de Ingeniería, Nicaragua, donde actualmente es docente y Gestor de Proyectos I+D+i. Desarrolló estudios de maestría en Ingeniería Ambiental en el Programa de Investigación, Estudios Nacionales y Servicios Ambientales (PIENSA-UNI). También posee un Master en Sistema de Información Geográfico por parte de la Universidad Católica San Antonio de Murcia, España (UCAM) y es un experto en ArcGIS certificado por ESRI España. Trabaja en línea de investigación vinculada con aguas residuales y SIG aplicado a la Hidrología y la recolección de residuos sólidos.

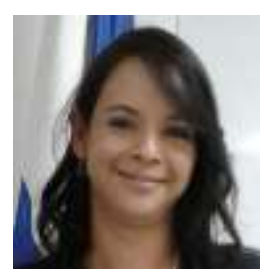

Rosa Verónica Reyes Rodríguez: Ingeniero Civil de la Universidad Nacional de Ingeniería, Nicaragua. Realizó estudios de Maestría en Ingeniera Ambiental en el Programa de Investigación, Estudios nacionales y Servicios Ambientales (PIENSA-UNI). Cuenta con 10 años de experiencia en diseño de Instalaciones hidrosanitarias y 6 años de experiencia en el diseño, instalación, inspección y mantenimiento de sistemas de protección contra incendio con énfasis en sistemas a base de agua. Está certificada en las Normas NFPA 13, 20, 25 y 101. Trabaja actualmente como Gerente de marcas en sistemas de protección contra incendio en el Grupo AquaCorp a nivel Centroamericano y Panamá. Tiene diferentes certificaciones con varias compañías fabricantes de bombas contra incendio. 